\title{
RELATIONSHIP BETWEEN YO-YO INTERMITTENT ENDURANCE TEST-LEVEL 1 AND MATCH RUNNING PERFORMANCE IN SOCCER: STILL ON THE RIGHT PATH?
}

\author{
TONI MODRIC, SIME VERSIC, DAMIR SEKULIC \\ University of Split, Faculty of Kinesiology, Split, Croatia
}

\author{
Mailing address: Damir Sekulic, University of Split, Faculty of Kinesiology, 6 Teslina Street, 21000 Split, Croatia, \\ e-mail: dado@kifst.hr
}

\begin{abstract}
Introduction. The Yo-Yo Intermittent Endurance Test-Level 1 (Yo-Yo IRl) is considered a valuable measurement tool in assessing specific soccer endurance. However, there is a lack of recent research validating this test with regard to match running performance (MRP) in elite-level soccer. Material and Methods. The aim of this study was to evaluate the validity of Yo-Yo IR1 in predicting MRP during elite-level soccer matches. The participants were soccer players who competed in the highest-level Croatian soccer league $(23.57 \pm 2.84$ years, $181.9 \pm 5.17 \mathrm{~cm}, 78.36 \pm 4.18 \mathrm{~kg})$, and they were all tested using Yo-Yo IR1 in the middle of the season 2019/2020. The players' MRP $(n=71)$ was measured using the Global Positioning System over one-half season, and included the total distance covered, the distance covered in different speed zones, accelerations and decelerations. The association between MRP and Yo-Yo IRl was identified with Pearson's correlation. Results. The results indicated that central midfielders and fullbacks achieved significantly higher results in Yo-Yo IR1 than forwards and central defenders (F-test: 29.80; $\mathrm{p}<0.01$; large effect size). Higher results in Yo-Yo IR1 were correlated with the match amount of (i) the total distance covered $(\mathrm{r}=0.65)$; (ii) high-speed running $(\mathrm{r}=0.42)$; (iii) high-intensity distance covered $(\mathrm{r}=0.36)$; (iv) total accelerations $(\mathrm{r}=0.37)$; and total decelerations $(\mathrm{r}=0.42)$. Conclusions. The established associations between Yo-Yo IRl and MRP in this study confirmed the validity of Yo-Yo IR1 in predicting MRP in professional soccer today.
\end{abstract}

Key words: aerobic fitness, specific endurance, field testing, football, running demands

\section{Introduction}

Soccer is physically high demanding sport characterized by high-intensity activities interspersed with periods of submaximal effort over an extended period of time [1, 2]. In particular, soccer players averagely cover $10-12 \mathrm{~km}$ in total during the game, while approximately $10 \%$ of this distance is covered at high intensities $[3,4]$. During the 90 minutes of the game, soccer players spend most of the time performing low to moderate intensity activities such as walking or jogging, and energy is mainly produced by aerobic metabolism $[4,5]$. Therefore, elite level soccer requires well-developed aerobic fitness $[6,7,8]$.

To assess aerobic fitness, soccer practitioners usually conduct different laboratory and field tests. In general, laboratory tests are traditionally considered the gold standard in evaluating aerobic fitness indicators - maximal oxygen consumption (VO2max), aerobic threshold (AeT) and anaerobic threshold (AnT) $[9,10]$. However, the results of laboratory assessments of aerobic fitness may have little relevance to soccer-specific training since such assessment requires exercise modes that are not common in soccer, such as linear running [11, 12]. Relatedly, recent studies have demonstrated very limited association between the aerobic fitness indicators obtained from laboratory testing and match running performance (MRP) $[8,10]$.

As an alternative to laboratory tests, field tests are widely accepted for the evaluation of aerobic fitness [11, 12, 13]. One of the most popular field tests focusing on the capacity to carry out intermittent exercise leading to maximal activation of the aerobic system is the Yo-Yo intermittent recovery test-level 1 (Yo-Yo IRI) [1]. In addition, Yo-Yo IRl has been shown to be a reliable, valid measurement of match-related fitness performance in intermittent sports such as soccer or basketball [14]. Naturally, the problem of association between Yo-Yo IRI and MRP has been the subject of many studies in recent years [15]. However, most of the studies have included young soccer players $[12,16]$, female players [17] or friendly matches [18], while there is a lack of studies that included elite-level players during official soccer matches.

Specifically, to the best of our knowledge, only one study has investigated MRP and Yo-Yo IRl among elite soccer players in official soccer matches where authors examined associations between Yo-Yo IRl and MRP, and reported a strong correlation $(\mathrm{r}=0.71)$ of the distance covered above a speed of $15 \mathrm{~km} / \mathrm{h}$ and Yo-Yo IRI [14]. However, the study of Krustrup and colleagues was conducted almost 20 years ago, and during that period, high-intensity activities in soccer matches have increased by $30-$ $35 \%$ [18]. Accordingly, we were of the opinion that the association between MRP and Yo-Yo IRl should be confirmed in a newly conducted study. Therefore, the main objective of this study was to examine the association between MRP and Yo-Yo IRl among elite soccer players during official soccer matches. Additionally, we analyzed the MRP and Yo-Yo IRl results according to specific playing positions.

\section{Material and Methods}

In this study we observed players that competed at the highest national competitive level. We tested their soccer-specific endurance using Yo-Yo IRI in the middle of the season 2019/2020, and analyzed their MRP over one half-season. This was done intentionally to minimize a possibility of changes in Yo-Yo IRl performance due to the training process. Yo-Yo IRI and MRP were evaluated according to the playing position. Afterwards, we examined the associations between Yo-Yo IRI and MRP. 


\section{Participants}

The participants $(\mathrm{n}=12)$ in this study were soccer players from Croatia $(\mathrm{M} \pm \mathrm{SD}$, age $23.57 \pm 2.84$ years, body height 181.9 $\pm 5.17 \mathrm{~cm}$, body mass $78.36 \pm 4.18 \mathrm{~kg}$ ), and they were all members of one team competing at the highest national level. The players were observed over half of one season 2019/2020, resulting in 71 MRPs that were used as cases for this study. Since team tactical formations did not include wingers (e.g., the team played in a 3-5-2 or 3-4-3 formation), players' MRPs (i.e., observations) were classified according to the playing position as central defenders $(n=14)$, fullbacks $(n=17)$, central midfielders $(n=29)$ and forwards $(n=11)$. All data were collected during 17 matches of the Croatian Soccer League 2019/2020 season. For the purposes of this study, only the results of those players who played for the entirety of a given match were analyzed. To minimize possible changes in Yo-Yo IRl performance, we did not analyze players who experienced injuries which excluded them from the training process for more than 2 weeks. Additionally, players that were not regular members of the squad were excluded from the analysis as well. All the participants signed an informed consent form agreeing to participate in this study. The study was approved by the Ethical Board of the Faculty of Kinesiology, Ubiversity of Split.

\section{Procedures}

The variables in this study included players' age, body height, weight, MRP and Yo-Yo IRl results. Anthropometrics were measured by an experienced evaluator using the standardized equipment (Seca, Birmingham, UK). MRP was measured during official matches and included the following: total distance covered $(\mathrm{m})$; distance covered in different speed categories: low-intensity running $(<14.3 \mathrm{~km} / \mathrm{h})$, high-speed running (19.8$25.1 \mathrm{~km} / \mathrm{h})$, sprinting $(\geq 25.2 \mathrm{~km} / \mathrm{h})$, and high-intensity running $(>19.8 \mathrm{~km} / \mathrm{h})$; total number (frequency) of accelerations $(>0.5$ $\left.\mathrm{m} / \mathrm{s}^{2}\right)$; number of high-intensity accelerations $\left(>3 \mathrm{~m} / \mathrm{s}^{2}\right)$; total number of decelerations (less than $-0.5 \mathrm{~m} / \mathrm{s}^{2}$ ); and the number of high-intensity decelerations (less than $-3 \mathrm{~m} / \mathrm{s}^{2}$ ). All MRP data were collected using GPS technology (S7 vector, Catapult, Melbourne, Australia) with a sampling frequency of $10 \mathrm{~Hz}$. The reliability and validity of this equipment have previously been described in detail [19].

Players were tested using the Yo-Yo IRl test on a soccer field in the middle of the season, before the winter preseason. All players performed the test after two light adaptation sessions to minimize the possibility of injury and the effect of fatigue. Just before the start of the test, players performed individual warm-up sessions consisting of 10 min of running at their own pace and 5 min of dynamic stretching. Players were instructed to run until voluntary exhaustion was reached and were given strong verbal encouragement throughout the test to elicit their best performance. Yo-Yo IRl consists of $2 \times 20 \mathrm{~m}$ shuttle runs at increasing speeds interspersed with 10 -second active recovery (controlled by audio signals from a compact disc player). Players run until they are not able to maintain the required speed, and the distance covered at that point is the test result [14]. The test was conducted in groups of a maximum of 8 players according to the above-described protocol.

\section{Statistics}

All data were log-transformed to reduce the nonuniformity of error, and normality was checked using the Kolmogorov-Smirnov test. The statistical analyses were performed on log-transformed data, but the results in tables and figures are presented as true-value means and standard deviations.
Differences in MRP and Yo-Yo IRl between playing positions were analyzed using one-way analysis of variance (ANOVA) with the Scheffe post hoc test. Effect size differences were established using ANOVA-derived partial eta squared (>0.02, small; > 0.13, medium; > 0.26, large) [20].

To identify the associations between MRP and Yo-Yo IRl, Pearson's correlation coefficients were calculated for the observed half-season period with the $\mathrm{r}$ coefficient classification as previously suggested: $\mathrm{r} \leq 0.35$ indicates a low or weak correlation, $\mathrm{r}=0.36$ to 0.67 indicates a moderate correlation, $\mathrm{r}=0.68$ to 1.0 indicates a strong or high correlation, and $r>0.90$ indicates a very high correlation [20]. For all analyses, Statistica (Version 13; TIBCO Software, Palo Alto, CA, USA) was used. A significance level of $\alpha=0.05$ was applied.

\section{Results}

Playing positions significantly differed in all MRP indices, with large ES difference for all variables, except high intensity accelerations (medium ES). The largest differences between playing positions were evidenced for total distance covered, with central midfielders achieving the highest values of all playing positions (significant post-hoc differences when compared to all other playing positions). Also, central midfielders dominated in the number of total decelerations (significant post-hoc differences when compared to all other playing positions), and the number of total accelerations (significant post-hoc differences when compared to central defenders and forwards). Forward players had the highest number of high intensity accelerations and decelerations, and dominated in sprinting (significant post-hoc differences where established when compared to central defenders and central midfielders), as well as in high speed running, and high intensity running (significant post-hoc differences in comparison to central midfielders).

Figure 1 presents descriptive statistics and playing-position differences in Yo-Yo IRl. Central midfielders and fullbacks achieved significantly higher results in Yo-Yo IRl than forwards and central defenders (F-test: 29.80; p < 0.01; large effect size). Specifically, central midfielders covered $1847 \mathrm{~m}$ in Yo-Yo IRl, while fullbacks covered $1840 \mathrm{~m}$. On the other hand, central defenders and forwards covered the lowest distance in Yo-Yo IRl (1434 $\mathrm{m}$ and $1520 \mathrm{~m}$, respectively).

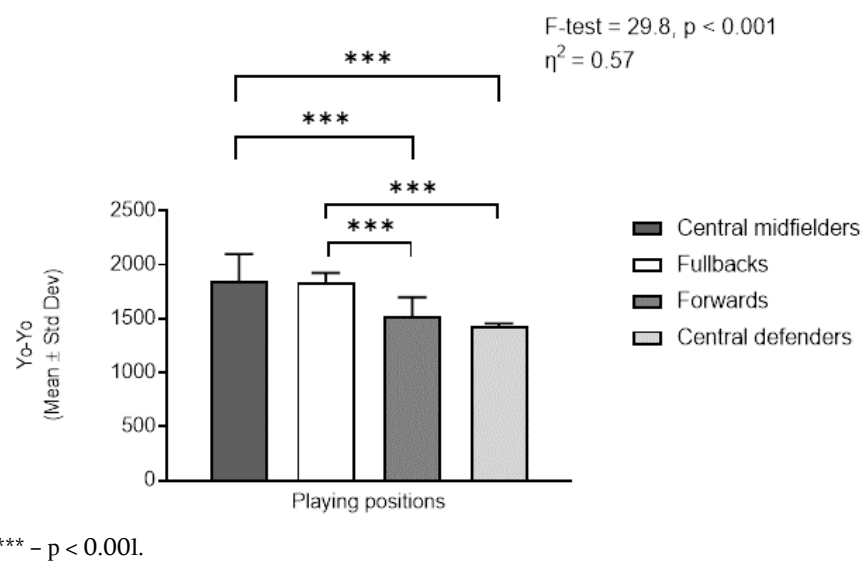

Figure 1. A Descriptive statistics of Yo-Yo IRl results, and differences between players in soccer-specific playing positions derived by the analysis of variance 
Table 1. Descriptive statistics of match running performance (data are given as Means \pm SD) and differences between players in soccerspecific playing positions derived by the analysis of variance

\begin{tabular}{|c|c|c|c|c|c|c|}
\hline & Central defenders & Fullbacks & Central midfielders & Forwards & F-test $(p)$ & $\eta^{2}$ \\
\hline Total distance covered (m) & $\begin{array}{c}9839.6 \pm 341.7 \\
\mathrm{CM}, \mathrm{FB}\end{array}$ & $\begin{array}{c}10812.6 \pm 647.4 \\
\mathrm{CM}, \mathrm{CD}\end{array}$ & $\begin{array}{c}11769.4 \pm 547.1 \\
\text { CD, FB, FW }\end{array}$ & $\begin{array}{c}10385.0 \pm 663.0 \\
C M\end{array}$ & $43.14(0.01)$ & 0.66 \\
\hline High speed running (m) & $\underset{C M}{393.1 \pm 111.9}$ & $\underset{\mathrm{CM}}{700.4 \pm 113.9}$ & $\begin{array}{c}636.8 \pm 161.6 \\
\mathrm{CD}, \mathrm{FB}, \mathrm{FW}\end{array}$ & $\underset{C M}{718.9 \pm 127.8}$ & $16.70(0.01)$ & 0.47 \\
\hline Sprinting (m) & $\begin{array}{c}91.0 \pm 46.9 \\
F B, F W\end{array}$ & $\begin{array}{c}222.8 \pm 96.4 \\
C M, C D\end{array}$ & $\begin{array}{c}96.7 \pm 55.7 \\
\quad F B, F W\end{array}$ & $\begin{array}{c}229.7 \pm 71.8 \\
C M, C D\end{array}$ & $20.53(0.01)$ & 0.36 \\
\hline High intensity distance (m) & $\begin{array}{c}484.0 \pm 151.9 \\
\mathrm{CM}, \mathrm{FB}\end{array}$ & $\begin{array}{c}923.0 \pm 165.6 \\
C M, C D\end{array}$ & $\begin{array}{c}733.4 \pm 205.5 \\
C D, F B, F W\end{array}$ & $9 \underset{\mathrm{CM}}{948.4 \pm 168.8}$ & $19.52(0.01)$ & 0.47 \\
\hline Total accelerations (rep) & $\underset{C M}{428.5 \pm 33.8}$ & $472.4 \pm 49.5$ & $\begin{array}{c}513.4 \pm 31.4 \\
C D, F W\end{array}$ & $\underset{\mathrm{CM}}{412.8 \pm 100.8}$ & $13.98(0.01)$ & 0.28 \\
\hline Total decelerations (rep) & $\underset{C M}{428.2 \pm 34.5}$ & $\underset{\mathrm{CM}}{461.4 \pm 47.8}$ & $\begin{array}{l}511.0 \pm 27.7 \\
\mathrm{CD}, \mathrm{FB}, \mathrm{FW}\end{array}$ & $\underset{C M}{435.1 \pm 43.8}$ & $21.16(0.01)$ & 0.47 \\
\hline High intensity accelerations (rep) & $\underset{F W}{16.2 \pm 8.5}$ & $23.1 \pm 10.2$ & $\begin{array}{c}21.4 \pm 8.4 \\
F W\end{array}$ & $\begin{array}{c}40.1 \pm 15.2 \\
C D, C M\end{array}$ & $12.67(0.01)$ & 0.20 \\
\hline High intensity decelerations (rep) & $\begin{array}{c}31.7 \pm 11.6 \\
F W\end{array}$ & $40.9 \pm 11.4$ & $\begin{array}{c}35.7 \pm 8.7 \\
F W\end{array}$ & $\begin{array}{c}54.5 \pm 10.2 \\
C D, C M\end{array}$ & $11.98(0.01)$ & 0.28 \\
\hline
\end{tabular}

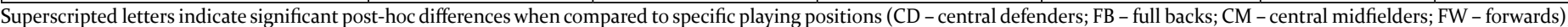
$\eta^{2}$ - partial eta squared.

Table 2. Correlations between match running performance and YoYo IRl distance covered

\begin{tabular}{|l|c|}
\hline & $\begin{array}{c}\text { Yo-Yo IR1 } \\
\text { Pearson's R }(\mathrm{p})\end{array}$ \\
\hline Total distance covered & $0.65(0.001)$ \\
\hline High speed running & $0.42(0.001)$ \\
\hline Sprinting & $0.17(0.16)$ \\
\hline High intensity distance & $0.36(0.01)$ \\
\hline Total accelerations & $0.37(0.01)$ \\
\hline Total decelerations & $0.42(0.001)$ \\
\hline High intensity accelerations & $-0.02(0.86)$ \\
\hline High intensity decelerations & $-0.04(0.74)$ \\
\hline
\end{tabular}

Table 2 presents correlations between MRP and distance covered in Yo-Yo IR1. Higher results in Yo-Yo IRl were correlated with the (i) total distance covered ( $r=0.65)$; (ii) high-speed running $(r=0.42)$; (iii) high-intensity distance covered $(r=0.36)$; and (iv) total accelerations $(\mathrm{r}=0.37)$ and total decelerations $(\mathrm{r}=0.42)$ in each match.

\section{Discussion}

The results from this study indicated moderate correlations between Yo-Yo IRl and total distance covered, high-intensity distance covered, the total number of accelerations and the total number of decelerations. In addition, the results indicated that of all playing positions, central midfielders and fullbacks reached the highest values in Yo-Yo IR1.

Yo-Yo IRI values in players in different playing positions

Although energy during a soccer match is mainly produced by aerobic metabolism, executing high-intensity movements (e.g., jumping, kicking, and even movements to mark the opponent) requires energy produced by anaerobic metabolism [22, $23,24]$. Therefore, there is an evident interest in the "simultane- ous" evaluation of both aerobic and anaerobic endurance, with the Yo-Yo IRl test being a very convenient and highly relevant field test since it assesses specific aerobic and anaerobic endurance for soccer [14].

Our study demonstrated that central midfielders and fullbacks achieved the highest values in Yo-Yo IRl (1846 $\mathrm{m}$ and 1840 $\mathrm{m}$, respectively). In particular, central midfielders are connection players between defense and attack [25] and to successfully participate in both directions of game play (e.g., defense and attack), they must possess a high level of both aerobic and anaerobic endurance. Similarly, although fullbacks are mainly defensive players, one of their main tactical duties is to perform crosses in the attacking phase of the game [26, 27]. To perform this repeatedly, they require a high level of both aerobic and anaerobic endurance.

These differences in Yo-Yo IRl values between playing positions are consistent with a study conducted on a sample of players from the Turkish TFF First League [28]. In that study, Bizati [28] investigated the physical and physiological characteristics of an elite Turkish soccer team according to playing positions and reported that wingers, fullbacks and central midfielders achieved the highest values in Yo-Yo IRl $(2515 \mathrm{~m}, 2475 \mathrm{~m}$ and $2465 \mathrm{~m}$, respectively), while central defenders and forwards averaged the lowest values (2195 $\mathrm{m}$ and $2305 \mathrm{~m}$, respectively). However, it is interesting to note that Yo-Yo IRl values of central midfielders and fullbacks from the previously cited Turkish study were approximately 30\% higher than the values from our study. We are of the opinion that the following explanations should be taken into account when considering these differences.

First, Turkish players were tested in the middle of the season that does not have a winter break [28]. Although our players were tested in the middle of the season as well, testing was basically conducted before the start of the winter preseason (e.g., the Croatian First Football League season is split into two halves, with a winter break of approximately 20 days between each half). Logically, the players' capacities are at a much higher level during the competitive part of the season (Turkish study) than in the preseason period (our study) [29]. Second, the Croatian First League is not physically highly demanding [30], and 
this can be reflected in the physical capacities of players who participate in the Croatian league.

\section{Correlations between Yo-Yo IRI and MRP}

We evidenced moderate correlations between Yo-Yo IRl and the total distance covered $(\mathrm{r}=0.65)$, high-speed running $(\mathrm{r}=$ $0.42)$, high-intensity distance covered $(\mathrm{r}=0.36)$, total number of accelerations $(\mathrm{r}=0.37)$ and total number of decelerations $(\mathrm{r}=0.42)$. Basically, players who achieve better results in Yo-Yo IRl will be able to handle MRP, which determines the volume of the game (e.g., total distance covered, total number of accelerations and total number of decelerations). Additionally, players with a higher level of specific endurance will be able to perform a greater amount of running at higher speeds. This is especially important because the amount of high-intensity running one can endure is considered one of the key elements for success in soccer $[30,31,32]$.

However, the association between high-intensity running and Yo-Yo IRl in our study was slightly lower than the same association presented by Krustrup et al. ( $r=0.37$ and 0.53 , respectively) [14]. Such findings can be explained by analyzing position-specific Yo-Yo IRl results. First, it must be mentioned that Krustrup et al. reported that fullbacks in their study achieved the highest values in Yo-Yo IRl out of all playing positions. At the same time, other studies confirmed that fullbacks cover a high amount of high-intensity distance [33, 34], which, taken together, may even result in a strong correlation between YoYo IRl and high-intensity running for the overall sample in the study carried out 20 years ago [14].

On the other hand, our study indicated that players who achieved the highest Yo-Yo IRl results played as central midfielders (see figure 1 for details). In general, players in this position do not cover a large high-intensity distance during soccer matches $[30,35]$. Thus, this factor decreases statistical possibilities of reaching stronger correlations between Yo-Yo IRl and high-intensity distance covered in our study.

It is interesting to note that the correlations between aerobic performance (measured with the field testing procedure) and MRP in our study were similar to those in studies where aerobic performance was measured in a laboratory setting [8]. In particular, a very recent study that analyzed associations between laboratory-derived aerobic indices and MRP evidenced a very low correlation between AnT and high-intensity distance covered $(r=0.28)$ and even no correlation between AeT and high-intensity distance covered [8]. Additionally, that study presented moderate correlations between AnT and AeT and the total distance covered $(\mathrm{r}=0.39$ and 0.45 , respectively). This is actually in accordance with the findings of the present study, in which we highlight moderate correlations between Yo-Yo IRl and both total distance covered and high-intensity distance covered $(r=0.65$ and 0.36 , respectively).

\section{Limitations and strengths}

The main limitation comes from the fact that this study focused on one team over half of one season only. Additionally, we only included players who (i) played the whole matches, (ii) did not have injuries that excluded them from the training process for more than 20 days, (iii) were regular members of the squad - which reduced the number of observations. In addition, Yo-Yo IRl testing was conducted after approximately 20 days of the break from the season, and the examined half-season lasted 20 weeks. As a result, it is possible that Yo-Yo IRl performance changed over half of the season due to the training process. This issue could directly affect the results of the association of MRP and Yo-Yo IR1.

This is one of the first studies to evaluate the validity of YoYo IRl by analyzing MRP of elite-level soccer players. Additionally, GPS and Yo-Yo IRl data were evaluated by the authors of the study, which reduces the possibility of data inconsistency.

\section{Conclusions}

Our results suggest that Yo-Yo IRl is applicable in endurance assessment in professional soccer. Moreover, given that YoYo IRl requires fewer resources than laboratory tests and is relatively simple, the authors of this study recommend using it in the assessment of soccer-specific endurance in elite male soccer players as a predictor of field running performance in matches. However, it must be noted that this study did not monitor changes in Yo-Yo IRl during the observed period and its possible influence on MRP. Therefore, future studies should analyze significant seasonal differences in Yo-Yo IRl performance when identifying association with MRP.

Our results demonstrated that soccer players who achieved better results in Yo-Yo IRl (e.g., possessed a higher level of specific endurance) could cover a greater total distance, perform a higher number of accelerations and decelerations, and cover more high-intensity distance during professional soccer matches. Therefore, these findings basically confirm the validity of YoYo IRl in predicting MRP.

Since better Yo-Yo IRl performance allows the handling of intermittent actions, we suggest that the conditioning process aimed at the development of specific endurance in soccer should include intermittent running drills that are similar to YoYo IRl running patterns. When creating these drills, differences in Yo-Yo IR1 results among players in different playing positions should be taken into account. For example, as we found the highest level of specific endurance (e.g., highest values in YoYo IRl) among central midfielders and fullbacks, these players should run at higher running speeds during running drills.

\section{References}

1. Bangsbo J., Iaia F.M., Krustrup P. (2008). The Yo-Yo intermittent recovery test. Sports Medicine 38(1), 37-51. DOI: 10.2165/00007256-200838010-00004.

2. Papadakis L., Tymvios C., Patras K. (2020). The relationship between training load and fitness indices over a pre-season in professional soccer players. The Journal of Sports Medicine and Physical Fitness 60(3), 329-337. DOI: 10.23736/ S0022-4707.20.10109-9.

3. Andrzejewski M., Chmura J., Pluta B., Konarski J.M. (2015). Sprinting activities and distance covered by top level Europa league soccer players. International Journal of Sports Science E Coaching 10(1), 39-50. DOI: 10.1260/1747-9541.10.1.39.

4. Paraskevas G., Hadjicharalambous M. (2018). Aerobic fitness of starter and non-starter soccer players in the Champion's League. Journal of Human Kinetics 61, 99-108. DOI: 10.1515/hukin-2017-0135.

5. Ingebrigtsen J., Dillern T., Shalfawi S.A. (2011). Aerobic capacities and anthropometric characteristics of elite female soccer players. The Journal of Strength $\mathcal{E}$ Conditioning Research 25(12), 3352-3357. DOI: 10.1519/ JSC.0b013e318215f763.

6. Castagna C., Impellizzeri F.M., Chaouachi A., Manzi V. (2013). Preseason variations in aerobic fitness and performance in elite-standard soccer players: a team study. The 
Journal of Strength E Conditioning Research 27(11), 29592965. DOI: 10.1519/JSC.0b013e31828d6la8.

7. Kozieł M., Adamczyk J.G. (2018). Changes in the level of motor preparation of professional soccer players in training. Polish Journal of Sport and Tourism 25(2), 14-19. DOI: 10.2478/pjst-2018-0009.

8. Modric T., Versic S., Sekulic D. (2021). Does aerobic performance define match running performance among professional soccer players? A position-specific analysis. Research in Sports Medicine 29(4), 336-348. DOI: 10.1080/15438627.2021.1888107.

9. Costill D.L. (1967). The relationship between selected physiological variables and distance running performance. The Journal of Sports Medicine and Physical Fitness 7(2), 61-66.

10. Metaxas T.I. (2021). Match running performance of elite soccer players: VO2max and players position influences. The Journal of Strength \& Conditioning Research 35(1), 162168. DOI: 10.1519/JSC.0000000000002646.

11. Castagna C., Chamari K., Stolen T., Wisloff U. (2005). Physiology of soccer: an update. Sports Medicine 35(6), 501-536. DOI: 10.2165/00007256-200535060-00004.

12. Castagna C., Manzi V., Impellizzeri F., Weston M., Alvarez J.C.B. (2010). Relationship between endurance field tests and match performance in young soccer players. The Journal of Strength $\mathcal{E}$ Conditioning Research 24(12), 3227-3233. DOI: 10.1519/JSC.0b013e3181e72709.

13. Lipecki K. (2018). Age-related differences in fitness performance and technical skills of young soccer players. Polish Journal of Sport and Tourism 25(4), 8-14. DOI: 10.2478/ pjst-2018-0021.

14. Krustrup P., Mohr M., Amstrup T., Rysgaard T., Johansen J. et al. (2003). The yo-yo intermittent recovery test: physiological response, reliability, and validity. Medicine $\mathcal{E}$ Science in Sports E Exercise 35(4), 697-705. DOI: 10.1249/01. MSS.0000058441.94520.32.

15. Schmitz B., Pfeifer C., Kreitz K., Borowski M., Faldum A. (2018). The Yo-Yo intermittent tests: a systematic review and structured compendium of test results. Frontiers in Physiology 9, 870. DOI: 10.3389/fphys.2018.00870.

16. Castagna C., Impellizzeri F., Cecchini E., Rampinini E., Alvarez J.C.B. (2009). Effects of intermittent-endurance fitness on match performance in young male soccer players. The Journal of Strength $\mathcal{E}$ Conditioning Research 23(7), 1954-1959. DOI: 10.1519/JSC.0b013e318lb7f743.

17. Krustrup P., Mohr M., Ellingsgaard H., Bangsbo J. (2005). Physical demands during an elite female soccer game: importance of training status. Medicine and Science in Sports and Exercise 37(7), 1242-1248. DOI: 10.1249/01. mss.0000170062.73981.94.

18. Barnes C., Archer D., Bush M., Hogg R., Bradley P. (2014). The evolution of physical and technical performance parameters in the English Premier League. International Journal of Sports Medicine 35(13), 1-6. DOI: 10.1055/s-0034-1375695.

19. Johnston R.J., Watsford M.L., Kelly S.J., Pine M.J., Spurrs R.W. (2014). Validity and interunit reliability of $10 \mathrm{~Hz}$ and $15 \mathrm{~Hz}$ GPS units for assessing athlete movement demands. The Journal of Strength E Conditioning Research 28(6), 1649-1655. DOI: 10.1519/JSC.0000000000000323.

20. Ferguson C.J. (2016). An effect size primer: A guide for clinicians and researchers. In A. E. Kazdin (ed.), Methodological issues and strategies in clinical research (pp. 301-310). American Psychological Association. DOI: 10.1037/14805-020.
21. Taylor R. (1990). Interpretation of the correlation coefficient: a basic review. Journal of Diagnostic Medical Sonography 6(1), 35-39. DOI: 10.1177/875647939000600106.

22. Modric T., Versic S., Sekulic D. (2020a). Aerobic fitness and game performance indicators in professional football players; playing position specifics and associations. Heliyon 6(11), e05427. DOI: 10.1016/j.heliyon.2020.e05427.

23. Redkva P.E., Paes M.R., Fernandez R., da-Silva S.G. (2018). Correlation between match performance and field tests in professional soccer players. Journal of Human Kinetics 62(1), 213-219. DOI: 10.1515/hukin-2017-0171.

24. Evangelos B., Lefteris M., Aristotelis G., Ioannis G., Natalia K. (2016). Aerobic and anaerobic capacity of professional soccer players in annual macrocycle. Journal of Physical Education and Sport 16(2), 527. DOI: 10.7752/jpes.2016.02083.

25. Clemente F.M., Martins F.M.L., Wong P.D., Kalamaras D., Mendes R.S. (2015). Midfielder as the prominent participant in the building attack: A network analysis of national teams in FIFA World Cup 2014. International Journal of Performance Analysis in Sport 15(2), 704-722. DOI: 10.1080/24748668.2015.11868778.

26. Van Lingen B. (1998). Coaching soccer: The official coaching book of the Dutch Soccer Association. Reedswain.

27. YiQ.,JiaH., Liu H., Gómez M.Á. (2018).Technicaldemandsof different playing positions in the UEFA Champions League. International Journal of Performance Analysis in Sport 18(6), 926-937. DOI: 10.1080/24748668.2018.1528524.

28. Bizati O. (2016). Physical and physiological characteristics of an elite soccer team's players according to playing positions. The Anthropologist 26(3), 175-180. DOI: 10.1080/09720073.2016.11892146.

29. Ferioli D., Bosio A., Zois J., La Torre A., Rampinini E. (2020). Seasonal changes in physical capacities of basketball players according to competitive levels and individual responses. PloS one 15(3), e0230558. DOI: 10.1371/journal. pone. 0230558 .

30. Modric T., Versic S., Sekulic D., Liposek S. (2019). Analysis of the association between running performance and game performance indicators in professional soccer players. International Journal of Environmental Research and Public Health 16(20), 4032. DOI: 10.3390/ijerph16204032.

31. Di Salvo V., Baron R., Tschan H., Montero F.C., Bachl N., Pigozzi F. (2007). Performance characteristics according to playing position in elite soccer. International Journal of Sports Medicine 28(03), 222-227. DOI: 10.1055/s-2006-924294.

32. Bradley P.S., Sheldon W., Wooster B., Olsen P., Boanas P., Krustrup P. (2009). High-intensity running in English FA Premier League soccer matches. Journal of Sports Sciences 27(2), 159-168. DOI: 10.1080/02640410802512775.

33. Rampinini E., Coutts A.J., Castagna C., Sassi R., Impellizzeri F. (2007). Variation in top level soccer match performance. International Journal of Sports Medicine 28(12), 1018-1024.

34. Modric T., Versic S., Sekulic D. (2020b). Playing position specifics of associations between running performance during the training and match in male soccer players. Acta Gymnica 50(2), 51-60. DOI: 10.5507/ag.2020.006.

35. Mallo J., Mena E., Nevado F., Paredes V. (2015). Physical demands of top-class soccer friendly matches in relation to a playing position using global positioning system technology. Journal of Human Kinetics 47(1), 179-188. DOI: 10.1515/ hukin-2015-0073.

Submitted: June 9, 2021

Accepted: September 23, 2021 